\title{
Sulphadoxine/pyrimethamine versus amodiaquine for treating uncomplicated childhood malaria in Gabon: A randomized trial to guide national policy
}

Basile Nsimba*1, Vincent Guiyedi², Modeste Mabika-Mamfoumbi², Jean Romain Mourou-Mbina ${ }^{2}$, Edgard Ngoungou ${ }^{2}$, Marielle Bouyou-Akotet ${ }^{2}$, Romaric Loembet ${ }^{2}$, Rémy Durand ${ }^{3}$, Jacques Le Bras ${ }^{4}$ and Maryvonne Kombila ${ }^{2}$

\begin{abstract}
Address: ${ }^{1}$ National Malaria Control Programme - Division for Disease Control, Ministry of Health, Brazzaville, Congo, ${ }^{2}$ Department of Parasitology-Mycology and Tropical Diseases, Faculty of Medicine and Health Sciences, Libreville, Gabon, ${ }^{3}$ Parasitology Laboratory, Avicenne Hospital, AP-HP, and EA 3406, Paris 13 University, Bobigny, France and ${ }^{4}$ National Malaria Reference Centre, Bichat-Claude Bernard Hospital, Paris, France, and EA 209, University Paris Descartes, France

Email: Basile Nsimba* - basilensimba@aol.com; Vincent Guiyedi - guidyvin@hotmail.com; Modeste Mabika-

Mamfoumbi - mabikmamfoumbi@yahoo.fr; Jean Romain Mourou-Mbina - mangondu20@yahoo.fr;

Edgard Ngoungou - ngoungou2001@yahoo.fr; Marielle Bouyou-Akotet - mariellebouyou@yahoo.fr; Romaric Loembet - rohmance@yahoo.fr; Rémy Durand - remy.durand@avc.aphp.fr; Jacques Le Bras - jacques.lebras@gmail.com; Maryvonne Kombila - valentine_favry@yahoo.fr

* Corresponding author
\end{abstract}

Published: 12 February 2008

Malaria Journal 2008, 7:31 doi:10.1 I86/I475-2875-7-3I

This article is available from: http://www.malariajournal.com/content/7/I/3I

(c) 2008 Nsimba et al; licensee BioMed Central Ltd.

This is an Open Access article distributed under the terms of the Creative Commons Attribution License (http://creativecommons.org/licenses/by/2.0), which permits unrestricted use, distribution, and reproduction in any medium, provided the original work is properly cited.
Received: 30 August 2007

Accepted: 12 February 2008

\begin{abstract}
Background: In Gabon, following the adoption of amodiaquine/artesunate combination (AQ/AS) as firstline treatment of malaria and of sulphadoxine/pyrimethamine (SP) for preventive intermittent treatment of pregnant women, a clinical trial of SP versus AQ was conducted in a sub-urban area. This is the first study carried out in Gabon following the WHO guidelines.

Methods: A random comparison of the efficacy of AQ (10 mg/kg/day $\times 3 \mathrm{~d})$ and a single dose of SP (25 $\mathrm{mg} / \mathrm{kg}$ of sulphadoxine/l.25 mg/kg of pyrimethamine) was performed in children under five years of age, with uncomplicated falciparum malaria, using the 28-day WHO therapeutic efficacy test. In addition, molecular genotyping was performed to distinguish recrudescence from reinfection and to determine the frequency of the dhps K540E mutation, as a molecular marker to predict SP-treatment failure.

Results: The day-28 PCR-adjusted treatment failures for SP and AQ were II.6\% (8/69; 95\% IC: 5.5-22.I) and $28.2 \%$ (20/7I; $95 \% \mathrm{Cl}$ : 17.7-38.7), respectively This indicated that SP was significantly superior to AQ $(P=0.019)$ in the treatment of uncomplicated childhood malaria and for preventing recurrent infections. Both treatments were safe and well-tolerated, with no serious adverse reactions recorded. The dhps $\mathrm{K} 540 \mathrm{E}$ mutation was not found among the 76 parasite isolates tested.

Conclusion: The level of AQ-resistance observed in the present study may compromise efficacy and duration of use of the AQ/AS combination, the new first-line malaria treatment. Gabonese policy-makers need to plan country-wide and close surveillance of AQ/AS efficacy to determine whether, and for how long, these new recommendations for the treatment of uncomplicated malaria remain valid.
\end{abstract}




\section{Background}

Chloroquine (CQ) resistance is widespread in Gabon and this forced the health authorities to revise the national malaria treatment policy $[1,2]$. In July 2003, following a national consensus meeting, the Gabonese Ministry of Health responded to WHO recommendations by adopting the amodiaquine/artesunate (AQ/AS) combination as first-line treatment, the artemether/lumefantrine $(\mathrm{A} / \mathrm{L})$ combination as second-line treatment, sulphadoxine/ pyrimethamine (SP) for preventing malaria in pregnant women; quinine remains the treatment of choice for severe malaria. Despite the considerable efforts of the Global Fund to help fighting malaria, the implementation of the WHO-recommended artemisinin-based combination treatments (ACTs) for controlling malaria encounters tremendous challenges in low-income countries, particularly in sub-Saharan Africa, because of the high cost of the drugs. Moreover, sound data supporting this new antimalarial-treatment policy is lacking in Gabon, and most in vivo studies to evaluate efficacy have been conducted with variable methodologies. Most previous studies were carried out in three provinces, namely Estuaire, MoyenOgooué and Haut-Ogooué, but none in Gabon's other six provinces. Consequently, studies carried out at the national level according to a standardized WHO protocol [3] were needed to determine the efficacy level of all antimalarial drugs to be used in the new national malaria treatment policy.

According to the data reported in the literature, Plasmodium falciparum CQ resistance was first reported in vitro in Gabon in 1983 [4] and confirmed by subsequent studies conducted throughout the country between 1984 and 1985 [5]. In 1987, CQ resistance was reported to have spread very fast, with an average reported rate of $42 \%$ [5]. Although this level of CQ resistance has remained stable since $[6,7]$, there was a need to change this treatment policy. The situation was similar in neighbouring countries [8-10]. It has been reported that an increase of infant mortality was attributable to the escalating $P$. falciparum resistance to CQ [11]. In Gabon, CQ has been used for many years as first-line drug for treating uncomplicated malaria and for preventing malaria. AQ and SP are the only cheap and widely available antimalarials and they were used together as second-line drugs before the change in drug policy. Regarding AQ efficacy, Gabon is an unusal case amongst Central African countries, because of regional differences. All studies carried out in Libreville (Estuaire province) demonstrated a good efficacy of $A Q$, with resistance rates below $15 \%[1,2,12]$, while those conducted in Lambarané (Haut-Ogooué province) and in Bakumba (Moyen-Ogooué province) reported unacceptable resistance rates, higher than $25 \%[13,14]$. A better understanding of the factors responsible for regional differences in AQ efficacy would be helpful to guide anew malaria treat- ment policy. The only previous studies assessing efficacy of SP were conducted in Franceville (Haut-Ogooué province) reporting a good efficacy of SP with clinical resistance rates below 10\% [15]. A clinical trial testing the efficacy of AS monotherapy conducted in Lambarané showed an excellent clinical efficacy with a PCR-corrected 28-day cure rate of $90 \%$ [16].

If the strategy of combining two or more antimalarial drugs is widely recommended as a means for improving treatment efficacy and delaying the development of resistance, a knowledge of the resistance level against individual components of the combination is essential. Policymakers in Gabon urgently need to know whether the newly-adopted malaria treatment strategy is a viable option in terms of therapeutic efficacy. They also need to learn how best to use information from clinical trials for optimizing the policy or considering when to make a further change in treatment policy.

The objectives of this study were to determine the efficacy level of AQ and SP using the standard WHO 28-day therapeutic efficacy test, with PCR-genotyping to distinguish recrudescences from reinfections due to therapeutic failures. Additionally, the prevalence of the dhps K540E mutation, previously defined as a predicting tool of SPresistance [17], was also assessed. This is the first such study conducted in Gabon using standard WHO protocols.

\section{Patients and Methods Study area}

Gabon is a Central African country bordering Equatorial Guinea and Cameroon to the north, and the Republic of Congo to the east. It has a $800 \mathrm{~km}$ coastline, providing access to the Atlantic Ocean to the west. It is situated on the equator and, hence, has a hot and humid climate, with precipitation nine months out of twelve. A dense forest covers $85 \%$ of its territory. Officially, Gabon's population is approximately 1.5 million, with $80 \%$ of the population living in towns. There are eight main ethnic groups, which are spread out across the country, but more than forty different ethnic groups altogether. On an administrative level, Gabon is subdivided into nine provinces, namely: Estuaire; Haut-Ogooué; Moyen Ogooué; Ngounié; Nyanga; Ogooué-Ivindo; Ogooué-Lolo; Ogooué-Maritime; and Woleu-Ntem. The rainy season is from October to May, and the dry season is from June to September. Malaria transmission is perennial with seasonal fluctuations.

\section{Study site}

This study was carried out between March-July 2005 in Oyem, a town of the Woleu-Ntem province of 35,000 inhabitants, near the northern borders with Cameroon 
and Equatorial Guinea, and $411 \mathrm{~km}$ away from Libreville (the political and administrative capital) to the southwest. Malaria is hyperendemic and transmission is perennial. In this forest region, where Anopheles gambiae s.s is considered to be the most important vector of malaria, the Plasmodium index in children under 15 years of age is ranging from $65 \%$ to $73 \%$, indicating an intense malaria transmission [18].

\section{Patient recruitment}

The study was conducted in fchildren with fever, below five years of age, attending the Out-patient Department of Oyem Hospital. Using the 28-day WHO therapeutic test for intense transmission areas of malaria [3], patients were enrolled in the study if they satisfied the following inclusion criteria: age between 6-59 months, mono-infection with $P$. falciparum, parasitaemia $\geq 2,000$ asexual parasites per $\mu \mathrm{L}$ of blood, free from severe malnutrition, absence of general danger signs or severe malaria, an axillary temperature of $37.5^{\circ} \mathrm{C}$ or above, absence of febrile conditions caused by diseases other than malaria, ability to come for the stipulated follow-up visits and easy access to the health facility, informed consent of parent/guardian, absence of history of hypersensitivity reactions to sulphonamides, and at least $5 \mathrm{~g}$ haemoglobin $(\mathrm{Hb}) / \mathrm{dL}$. Neither a history of previous antimalarial drug use, nor the presence of antimalarial drugs in the urine was an exclusion criterion in the WHO standard protocol $[3,19]$. Before enrolment in the study, a medical history of each patient was obtained from their accompanying parent or guardian and the child was clinically examined by a physician. Body weight and axillary or ear temperature were recorded; thick and thin smears were Giemsa-stained (5\% Giemsa $\mathrm{R}$ for $20 \mathrm{~min}$.) for parasite identification and quantification. Parasitaemia (parasites/ $\mu \mathrm{L}$ ) was measured by counting the number of asexual parasites against 200 leucocytes in the Giemsa-stained thick blood smears, based on a mean count of 8,000 leucocytes per $\mu \mathrm{L}$ of blood. A slide was declared negative only after microscopic fields corresponding to at least 500 leucocytes had been checked. Two experienced technicians performed the microscopy independently, each time comparing their results. The principal investigator closely supervised the study team to ensure consistency and accuracy of the data. Some slides randomly chosen were re-read in the laboratory in Paris.

Sample size estimations for the therapeutic test were performed by assuming that the treatment failures rate would be significantly less than $15 \%$ for SP and AQ, referring to previous clinical trials outcomes. Thus, according to WHO recommendations, a minimum of 50 patients was required for each therapeutic group [3].

\section{Patient treatment and follow-up}

After obtaining informed consent from parents or guardians on day 0 , the enrolled children were randomly allotted to one of two treatment groups, to receive either AQ base (Camoquin ${ }^{\circledR}$ tablets, Pfizer, Dakar, Senegal) $30 \mathrm{mg} /$ $\mathrm{kg}$ body weight over three days (i.e. $10 \mathrm{mg} / \mathrm{kg}$ daily), or SP (Maloxine $^{\circledast}$ tablets, Exphar, Belgium) as a single dose of 25 $\mathrm{mg} / \mathrm{kg}$ of sulphadoxine $/ 1.25 \mathrm{mg} / \mathrm{kg}$ of pyrimethamine.

An antipyretic (paracetamol, $15 \mathrm{mg} / \mathrm{kg}$, every $8 \mathrm{~h}$ for $24 \mathrm{~h}$ ) was systematically given on day 0 and if needed on day 1 and 2. All tablets were administered orally by a nurse in the presence of the physician. For 30 min following drug administration, patients were observed for vomiting and other side-effects. The same dose was re-administered if vomiting occurred. On days 1 and 2, symptoms, other medications, temperature, and physical examination were recorded, but microscopy was not performed unless one or more of danger signs were present. The same clinical observation was repeated and parasitological examination was conducted on days 3, 7, 14, 21 and 28. On day 0 , when the patient presented a fever without parasitaemia in the absence of another pathology the child was seen on the next day for intensive follow-up and microscopic diagnosis of malaria. All treatment failures were treated with quinine tablets $(8 \mathrm{mg} / \mathrm{kg}$ base three times daily for seven days).

\section{Classification of treatment outcomes}

The responses to drug treatment were classified according to the WHO protocol [3], as an adequate clinical and parasitological response (ACPR), early treatment failure $(\mathrm{ETF})$, and late treatment failure (LTF), including late clinical failure (LCF) and late parasitological failure (LPF).

\section{Molecular genotyping of parasite isolates}

Finger-prick blood samples blotted on Isocode ${ }^{\circledR}$ filter papers (Schleicher and Schuell, Ecquevilly, France) were dried and stored at room temperature in small separated and sealed plastic bags, prior to genotyping analysis at the National Malaria Reference Centre, Bichat-Claude Bernard Hospital, Paris, France. DNA was extracted with chelex-100 resin as previously reported [20]. Merozoite surface protein-2 (msp-2), which has been shown to be sufficiently discriminating in African parasite populations [21], was amplified by PCR for paired pre-treatment and failure samples to distinguish recrudescences from reinfections, as previously described [22]. Genotyping results PCR-adjusted were classified into three categories: 1) true recrudescences, 2) reinfections, and 3) mixed results (recrudescence and reinfection). Treatment outcomes with PCR correction were based on the number of recrudescences and reinfections, as indicated elsewhere [23]. The cases of mixed results were considered as recrudescences, and unconclusive results (PCR-DNA amplification 
failure) were excluded from the analysis. In addition, PCR-genotyping followed by sequencing method, as previously reported [17], was performed for assessing the prevalence of $d h p s$ K540E mutation.

\section{Ethical considerations}

The local health and institutional authorities approved the research protocol (Gabonese Ministry of Public Health). Verbal and written informed consent for participation were obtained from parents or guardians, after thorough information on the study was provided in the local language.

\section{Statistical analysis}

Data were analysed using version 2000 of the Epi-info software (Centers for Disease Control and Prevention, Atlanta, GA) and Graphpad Instat software (Graph Pad software, 10855 Sorrento Valley Road \#203, San Diego CA92121 USA). Proportions were compared by performing Fisher's Exact test. The therapeutic response at day-28 of follow-up with its corresponding 95\% confidence intervals was calculated using an intention-to-treat analysis, which included all patients who fulfilled enrolment criteria. Standard deviation (SD) was generally indicated for means and $P$-values of $<0,05$ were calculated to demonstrate differences statistically.

\section{Results}

Of the 632 febrile children with suspected malaria who were screened, three hundred-two $(47.8 \% ; 302 / 632)$ had confirmed malaria, of whom 154 met the inclusion criteria. Three hundred forty-four $(54.4 \% ; 344 / 632)$ were male; their mean age was 32.2 months.

Of the 154 enrolled children, 78 were randomly assigned to AQ and 76 to SP. No difference was observed between the two therapeutic groups in terms of gender, mean age, mean body weight, presence of documented fever (axillary temperature $\geq 37.5^{\circ} \mathrm{C}$ ) and parasitaemia. Overall, fourteen patients were excluded or lost to follow up (seven in AQ group and seven in SP group) during the follow-up period because of failure to follow the protocol (antimalarial treatment administered by themselves or a third party) or failure to come for follow-up on the scheduled days (generally because of travel for several days outside the city). Details of the patient follow-up are reported in Figure 1 (trial profile). No severe adverse drug reactions were observed during the follow-up of the patients in any therapeutic group. The day-28 PCR-adjusted failure rates were significantly higher in the AQ $(28.2 \% 20 / 71 ; 95 \%$ CI: $17.7-38.7)$ group than in the SP group $(11.6 \% 8 / 69$; 95\% CI: 5.5-22.1), $(P=0.0195)$. Of the 32 late treatment failures recorded in AQ group, detailed PCR-genotyping results were as following: 13 recrudescences, six reinfections, seven mixed results (recrudescence + reinfection) and six unconclusive results (PCR-amplification failure). Regarding SP group, detailed PCR-genotyping results among 14 late treatment failures were as following: eight true recrudescences and six reinfections. SP was superior to AQ for preventing recurrent infections (Table 1). The dhps K540E mutation was not found among the 76 analysed blood samples from patients allocated to the SP group.

\section{Discussion}

The results of this study show, both clinically and parasitologically, that SP is significantly superior to AQ in terms of efficacy, with day-28 PCR-corrected failure rates of $11.6 \%$ and $28.2 \%$ respectively. SP also was more effective than AQ for preventing recurrent infections. Both regimens were safe and well-tolerated, with no serious adverse reaction recorded during the course of the study. Although protocols of previous studies were not in conformity with the standard WHO protocol, our data concerning SP efficacy are consistent with those reported in other parts of the country, such as in Bakoumba (HautOgooué province) indicating a treatment failure rate of $14 \%$ at day 28 without PCR-adjustment in children under 10 years [14]. A good efficacy of SP was also observed in neighbouring Congo, Cameroon and Equatorial Guinea $[9,10,24]$. The absence of the dhps K540E mutation observed in the present study was already described in Gabon [25] and in neighbouring countries $[17,25,26]$. There is now a clear evidence that the principal role attributed to the dhps K540E mutation as a molecular marker predicting clinical SP-treatment failures is not true either in the existing literature or in the present study where $11.6 \%$ of SP resistance was found without a single instance of dhps K540E mutation. The AQ-resistance level reported in the study is too high, according to WHO guidelines (AQ-treatment failures $>25 \%$ ). The present results regarding AQ efficacy are consistent with other studies from Lambarané (Moyen-Ogooué province) and Bakumba (Haut-Ogooué province), reporting worse AQ efficacy with PCR-uncorrected resistance rates ranging from $34.7 \%$ to $47 \%$ on day $28[13,14]$. In contrast, the results from Libreville (Estuaire Province) have reported low AQ-failure rates ranging from $0 \%$ to $13 \%$ on day 14 $[1,2,12]$. Though these data are lacking PCR-adjustment, it seems that $\mathrm{AQ}$ efficacy is creating geographic differences in Gabon. Further AQ efficacy studies carefully carried out according to standard WHO protocol in other parts of the country, including settings reporting high AQ-failure rates, are urgently needed. Geographic differences of AQ efficacy cannot be directly explained by differences in malaria transmission levels between different regions of the country, because it was reported that transmission intensity does not directly affect the evolution of drugresistance [27]. However, the relative importance of immunity in determining the response to therapy has 


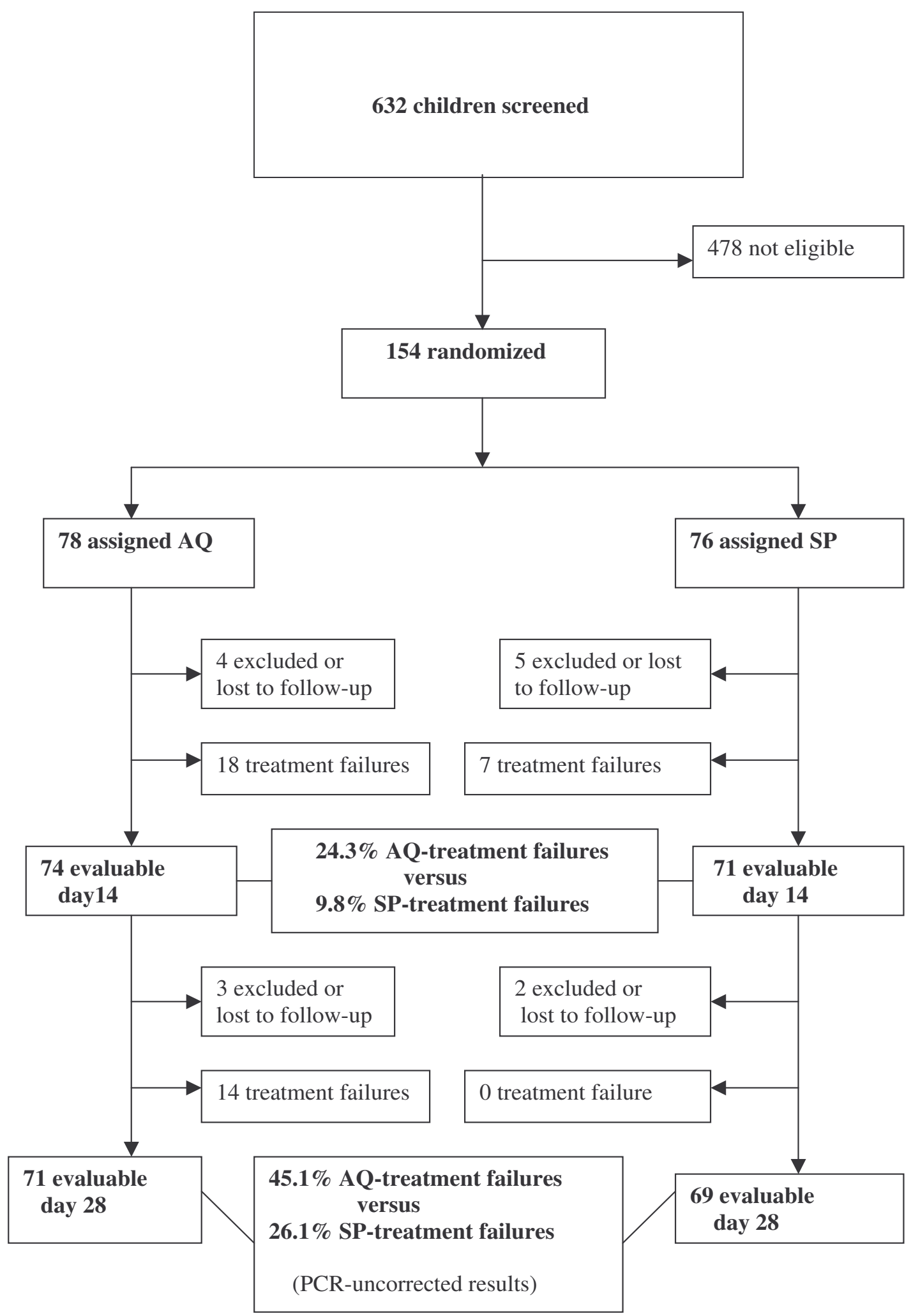

Figure I

Trial Profile. $A Q=$ amodiaquine; $S P$ = sulphadoxine/pyrimethamine. 
Table I: Efficacy at day-28 of sulphadoxine/pyrimethamine (SP) versus amodiaquine (AQ)

\begin{tabular}{|c|c|c|c|c|c|c|c|}
\hline & \multicolumn{3}{|c|}{ SP } & \multicolumn{3}{|c|}{$\mathrm{AQ}$} & \multirow[b]{2}{*}{$P$ value } \\
\hline & $\%$ & $\mathrm{n}$ & $95 \% \mathrm{Cl}$ & $\%$ & $\mathrm{n}$ & $95 \% \mathrm{Cl}$ & \\
\hline ACPR & 73.9 & 51 & $61.7-83.4$ & 54.9 & 39 & $42.7-66.6$ & 0.03 \\
\hline ETF & 2.9 & 2 & $0.5-11.0$ & 0 & 0 & $0.0-6.4$ & 0.46 \\
\hline LCF & 1.4 & I & $0.2-7.8$ & 5.6 & 4 & $1.8-14.5$ & 0.38 \\
\hline LPF & 21.7 & 15 & $13.1-33.6$ & 39.4 & 28 & $28.3-51.8$ & 0.04 \\
\hline \multicolumn{8}{|l|}{ Failure rate } \\
\hline Before PCR-correction & 26.1 & $18 / 69$ & $15.7-36.5$ & 45.1 & $32 / 71$ & $33.4-57.3$ & 0.02 \\
\hline After PCR-correction & 11.6 & $8 / 69$ & $5.5-22.1$ & 28.2 & $20 / 71$ & $17.7-38.7$ & 0.019 \\
\hline
\end{tabular}

$\mathrm{ACPR}=$ adequate clinical and parasitological response; $\mathrm{ETF}=$ early treatment failure; $\mathrm{LTF}=$ late treatment failure; $\mathrm{LCF}=$ late clinical failure; $\mathrm{LPF}=$ late parasitological failure

been demonstrated in other studies, which had shown that age, a surrogate marker of acquired immunity, is associated with the ability to clear parasites with resistant genotypes [28]. It has been reported that cytochrome P450 CYP2C8 is involved in the metabolism of $\mathrm{AQ}$, and additionally some information is currently available concerning its variation among ethnic groups as in Zanzibar and in Ghana $[29,30]$; this also needs to be investigated in Gabon in order to understand these inter-provincial differences of AQ efficacy.

In conclusion, geographical differences of AQ efficacy discussed in this study are a major concern in Gabon now that the AQ/AS combination has been selected as the new malaria first-line treatment policy. Thus, further investigations carefully conducted in the respect of good clinical practices are necessary to ascertain AQ-resistance at the national level.

In a country where ACTs are implemented, classical antimalarial drugs, including AQ and SP, must be monitored by recruiting persons with asymptomatic $P$. falciparum infection. The results of this longitudinal prospective study will provide an essential baseline of the parasitological efficacy of those monotherapies and also to be able to understand, at a later stage, the natural history of the evolution of resistance to ACTs.

\section{Authors' contributions}

$\mathrm{BN}, \mathrm{VG}, \mathrm{RD}, \mathrm{JLB}$ and MK designed the study and contributed to the discussion. MMM, JRMM, EN, MBA and RL participated to the clinical study. JRMM, BN, RD processed samples and analysed the data. $\mathrm{BN}$ wrote the first draft of the manuscript, then VG, RD, JLB and MK critically reviewed the manuscript. All authors read and approved the final manuscript.

\section{Conflict of interest}

The author(s) declare that they have no competing interests.

\section{Acknowledgements}

We are grateful to local health authorities for their contribution to this study. We are very thankful to Nestor Obiang Bekale for his strong technical contribution. We thank the communities of Oyem for their patience and participation in the trial, and thank the health staff of the Oyem Hospital for their cooperation. We thank Veronique Hubert for doing molecular typing. We also thank Dr Pascal Ringwald (TDR, WHO) for his invaluable advice. Financial support was provided by the Institute of African Medicine and Epidemiology (IMEA, Fondation Léon Mba, Paris).

\section{References}

I. Guiyedi V, Koko J, Bouyou Akotet M, Mabika Mamfoumbi M, Matsiegui PB, Traore B, Kombila M: Evaluation of efficacy and tolerance of amodiaquine versus chloroquine in the treatment of uncomplicated malaria outbreak in children of Gabon. Bull Soc Pathol Exot 200I, 94:253-257.

2. Brasseur P, Guiguemde R, Diallo S, Guiyedi V, Kombila M, Ringwald $P$, Olliaro P: Amodiaquine remains effective for treating uncomplicated malaria in west and central Africa. Trans $R$ Soc Trop Med Hyg 1999, 93:645-650.

3. WHO: Assessment of therapeutic efficacy of antimalarial drugs for uncomplicated falciparum malaria in areas with intense transmission Geneva, Switzerland: World Health Organization; 2003. WHO/HTM/RBM/ 2003.50

4. Burchard GD, Horstmann RD, Wernsdorfer WH, Dietrich M: Plasmodium falciparum malaria: resistance to chloroquine, but sensitivity to mefloquine in the Gabon. A prospective invitro study. Tropenmed Parasitol 1984, 35: I-4.

5. Richard-Lenoble D, Kombila M, Martz M, Lefevre B, Chandenier J, Gay F, Billiault X, Therizol-Ferly M: Development of chloroquine resistance in Plasmodium falciparum in Gabon between 1984 and 1987-88 (in vivo evaluation in a school environment). Ann Soc Belg Med Trop 1989, 69:1 I3-119.

6. Gueret D, Migot F, Ringwald P, Thibaut P, Le Bras J: Stability of Plasmodium falciparum resistance to chloroquine between 1987 and 1989 in Mounana, Gabon. Bull World Health Organ 1992, 70:621-624.

7. Pradines B, Mabika Mamfoumbi M, Keundjian A, Lebeau C, Fusai T, Owono Medang M, Rogier C, Parzy D, Kombila M: In vitro sensitivity of Plasmodium falciparum isolates from Gabon to chloroquine and cycloguanil. Bull Soc Pathol Exot 1999, 92:91-94.

8. Ringwald P, Same Ekobo A, Keundjian A, Kedy Mangamba D, Basco LK: Chemoresistance of $P$. falciparum in urban areas of Yaounde, Cameroon. Part I: Surveillance of in vitro and in vivo resistance of Plasmodium falciparum to chloroquine from 1994 to 1999 in Yaounde, Cameroon. Trop Med Int Health 2000, 5:612-619.

9. Roche J, Guerra-Neira A, Raso J, Benito A: Surveillance of in vivo resistance of Plasmodium falciparum to antimalarial drugs from 1992 to 1999 in Malabo (Equatorial Guinea). Am J Trop Med Hyg 2003, 68:598-60I.

10. Nsimba B, Malonga DA, Mouata AM, Louya F, Kiori J, Malanda M, Yocka D, Oko-Ossho J, Ebata-Mongo S, Le Bras J: Efficacy of sul- 
phadoxine/pyrimethamine in the treatment of uncomplicated Plasmodium falciparum malaria in Republic of Congo. Am J Trop Med Hyg 2004, 70:133-138.

II. Trape JF: The public health impact of chloroquine resistance in Africa. Am J Trop Med Hyg 200I, 64:12-17.

12. Adjuik M, Agnamey P, Babiker A, Borrmann S, Brasseur P, Cisse M, Cobelens F, Diallo S, Faucher JF, Garner P, Gikunda S, Kremsner PG, Krishna S, Lell B, Loolpapit M, Matsiegui PB, Missinou MA, Mwanza J, Ntoumi F, Olliaro P, Osimbo P, Rezbach P, Some E, Taylor WR: Amodiaquine-artesunate versus amodiaquine for uncomplicated Plasmodium falciparum malaria in African children: a randomised, multicentre trial. Lancet 2002, 359: I365-I372.

13. Borrmann S, Faucher JF, Bagaphou T, Missinou MA, Binder RK, Pabisch S, Rezbach P, Matsiegui PB, Lell B, Miller G, Kremsner PG: Atovaquone and proguanil versus amodiaquine for the treatment of Plasmodium falciparum malaria in African infants and young children. Clin Infect Dis 2003, 37: |44|-| 447

14. Aubouy A, Bakary M, Keundjian A, Mbomat B, Makita JR, MigotNabias F, Cot M, Le Bras J, Deloron P: Combination of drug level measurement and parasite genotyping data for improved assessment of amodiaquine and sulfadoxine-pyrimethamine efficacies in treating Plasmodium falciparum malaria in Gabonese children. Antimicrob Agents Chemother 2003, 47:23I-237.

15. Deloron P, Mayombo J, Le Cardinal A, Mezui-Me-Ndong J, BruziBaert C, Lekoulou F, Elissa N: Sulfadoxine-pyrimethamine for the treatment of Plasmodium falciparum malaria in Gabonese children. Trans R Soc Trop Med Hyg 2000, 94:188-190.

16. Schwarz NG, Oyakhirome S, Potschke M, Glaser B, Klouwenberg PK, Altun H, Adegnika AA, Issifou S, Kun JF, Kremsner PG, Grobusch MP: 5-day nonobserved artesunate monotherapy for treating uncomplicated Falciparum malaria in young Gabonese children. Am J Trop Med Hyg 2005, 73:705-709.

17. Nsimba B, Jafari-Guemouri S, Malonga DA, Mouata AM, Kiori J, Louya $F$, Yocka D, Malanda M, Durand R, Le Bras J: Epidemiology of drugresistant malaria in Republic of Congo: using molecular evidence for monitoring antimalarial drug resistance combined with assessment of antimalarial drug use. Trop Med Int Health 2005, 10:1030-1037.

18. Richard-Lenoble D, Kombila M, Chandenier J, Gay F, Billiault X, Nguiri C, Martz M, Boyer F, Bauzou M: Malaria in Gabon. 2. Evaluation of the qualitative and quantitative prevalence of parasites in the total school and preschool population of the country. Bull Soc Pathol Exot 1987, 80(3 Pt 2):532-542.

19. WHO: Assessment of therapeutic efficacy of antimalarial drugs for uncomplicated falciparum malaria in areas with intense transmission Geneva, Switzerland: World Health Organization; 1996. WHO/MAL/96.1077

20. Plowe CV, Djimdé A, Bouare M, Doumbo OK, Wellems TE: Pyrimethamine and Proguanil resistance-conferring mutations in Plasmodium falciparum dihydrofolate reductase polymerase chain reaction methods for surveillance in Africa. Am J Trop Med Hyg 1995, 52:565-568.

21. Slater M, Kiggundu M, Dokomajilar C, Kamya MR, Bakyaita N, Talisuna $A$, Rosenthal PJ, Dorsey G: Distinguishing recrudescences from new infections in antimalarial clinical trials: major impact of interpretation of genotyping results on estimates of drug efficacy. Am J Trop Med Hyg 2005, 73:256-262.

22. Jafari S, Durand R, Lusina D, Le Bras J: Molecular characterisation of airport malaria: four cases in France during summer 1999. Parasite 2002, 9:187-19|.

23. Stepniewska $K$, White N: Some considerations in the design and interpretation of antimalarial drug trials in uncomplicated falciparum malaria. Malar J 2006, 5:I27.

24. Basco LK, Ngane VF, Ndounga M, Same-Ekobo A, Youmba JC, Abodo RT, Soula G: Molecular epidemiology of malaria in Cameroon. XXI. Baseline therapeutic efficacy of chloroquine, amodiaquine, and sulfadoxine-pyrimethamine monotherapies in children before national drug policy change. Am J Trop Med Hyg 2006, 75:388-395

25. Aubouy A, Jafari S, Huart V, Migot-Nabias F, Mayombo J, Durand R, Bakary M, Le Bras J, Deloron P: DHFR and DHPS genotypes of Plasmodium falciparum isolates from Gabon correlate with in vitro activity of pyrimethamine and cycloguanil, but not with sulfadoxine-pyrimethamine treatment efficacy. J Antimicrob Chemother 2003, 52:43-49.
26. Basco LK, Tahar R, Keundjian A, Ringwald P: Sequence variations in the genes encoding dihydropteroate synthetase and dihydrofolate reductase and clinical response to sulfadoxinepyrimethamine in patients with acute uncomplicated falciparum malaria. J Infect Dis 2000, 182:624-628.

27. Hastings IM, Watkins WM: Intensity of malaria transmission and the evolution of drug resistance. Acta Trop 2005, 94:2। 8-229.

28. Dorsey G, Gasasira AF, Machekano R, Kamya MR, Staedke SG, Hubbard $A$ : The impact of age, temperature, and parasite density on treatment outcomes from antimalarial clinical trials in Kampala, Uganda. Am J Trop Med Hyg 2004, 7 I:53 I-536.

29. Cavaco I, Strömberg-Nörklit J, Kaneko A, Msellem MI, Dahoma M, Ribeiro VL, Bjorkman A, Gil JP: CYP2C8 polymorphism frequencies among malaria patients in Zanzibar. Eur J Clin Pharmacol 2005, 61: : 15-18.

30. Röwer S, Bienzle U, Weise A, Lambertz U, Forst T, Otchwemah RN, Pfützner A, Mockenhaupt FP: High prevalence of the cytochrome P450 2C8*2 mutation in Northern Ghana. Trop Med Int Health 2005, 10:1271-1273.
Publish with Bio Med Central and every scientist can read your work free of charge

"BioMed Central will be the most significant development for disseminating the results of biomedical research in our lifetime. "

Sir Paul Nurse, Cancer Research UK

Your research papers will be:

- available free of charge to the entire biomedical community

- peer reviewed and published immediately upon acceptance

- cited in PubMed and archived on PubMed Central

- yours - you keep the copyright 